\title{
INFECTION AND APOPTOSIS ASSOCIATED WITH INFLAMMATION IN PERIODONTITIS:
} AN IMMUNOHISTOLOGIC STUDY

\author{
Dyah Listyarifah $^{1,2}$, Ahmed Al-Samadi ${ }^{3}$, Abdelhakim Salem ${ }^{1,3}$, Ahmad Syaify ${ }^{4}$, Tuula Salo ${ }^{3,5}$, Taina \\ Tervahartiala $^{3}$, Daniel Grenier ${ }^{6}$, Dan C. Nordström ${ }^{7}$, Timo Sorsa ${ }^{3,8}$, Mari Ainola ${ }^{1}$ \\ ${ }^{1}$ Department of Medicine, Clinicum, University of Helsinki, and Helsinki University Central Hospital, \\ Helsinki, Finland \\ ${ }^{2}$ Department of Dental Biomedical Sciences, Faculty of Dentistry, Universitas Gadjah Mada, Indonesia \\ ${ }^{3}$ Department of Oral and Maxillofacial Diseases, University of Helsinki, and Helsinki University Central \\ Hospital, Helsinki, Finland \\ ${ }^{4}$ Department of Periodontology, Faculty of Dentistry, Universitas Gadjah Mada, Indonesia \\ ${ }^{5}$ Oulu University Central Hospital, Institute of Dentistry, University of Oulu, Department of Diagnostics and \\ Oral Medicine, Oulu, Finland \\ ${ }^{6}$ Oral Ecology Research Group, Faculty of Dentistry, Université Laval, Quebec, Canada \\ ${ }^{7}$ Department of Internal Medicine and Rehabilitation, University of Helsinki, and Helsinki University Central \\ Hospital, Helsinki, Finland \\ ${ }^{8}$ Division of Periodontology, Department of Dental Medicine, Karolinska Institutet, Huddinge, Sweden
}

\section{Correspondence address:}

Dyah Listyarifah, MD., MSc.

Biomedicum Helsinki 1 /4th floor

Clinicum, Faculty of Medicine

University of Helsinki

Haartmaninkatu 8

00290 Helsinki. Finland

Mobile +358-46-5402104

E-mail: dyah.listyarifah@helsinki.fi 


\begin{abstract}
OBJECTIVE: Evidence of increased apoptosis is observed in periodontitis, and may be associated with destruction of the periodontal tissue caused by the increased cell death, with the release of danger signals and subsequent stimulation of the proinflammatory processes. However, the exact mechanisms associated with these processes remain unclear. This study aimed to investigate the presence of the periodontal pathogen Treponema denticola, apoptosis, High Mobility Group Box1 as a damage-associated molecular pattern and several inflammatory markers in periodontitis and gingivitis subjects.

MATERIALS AND METHODS: Soft tissue specimens from gingival tissues of periodontitis and gingivitis patients were used for immunohistochemical and immunofluorescence staining of Treponema denticola chymotrypsin-like proteinase (CTLP), apoptosis markers, High Mobility Group Box1, Toll-like receptor 4, inflammatory cell markers and proinflammatory cytokines.
\end{abstract}

RESULTS: Treponema denticola was detected in all periodontitis-affected tissues. This was associated with a significant increase in the number of apoptotic cells, including macrophages, alterations in the expression of High Mobility Group Box 1 and its receptor, and increased levels of proinflammatory cytokines compared with gingivitis.

CONCLUSIONS: In summary, the presence of Treponema denticola (especially its CTLP), apoptosis, High Mobility Group Box1, and inflammatory markers suggest their potential involvement in the pathogenesis of periodontitis.

Keywords: apoptosis; HMGB1 protein; inflammation; periodontitis

Running title: HMGB1 and inflammation in periodontitis 


\section{Introduction}

Periodontitis is an inflammatory disorder of bacterial origin that leads to destruction of soft and hard periodontal tissues and subsequently leading to tooth loss (Page et al, 1997; Sorsa et al, 2006). This disease is induced by a limited number of bacteria found in the subgingival biofilm. In this study, we focused on Treponema denticola, which represents one of the most important pathogen in periodontitis. As a matter of fact, $T$. denticola has been strongly associated with the severity of periodontitis (Riviere et al, 1997; Sela, 2001). T. denticola adheres to the host tissue and expresses a chymotrypsin-like proteinase (CTLP), which can degrade various structural proteins of the gingiva. Consequently, $T$. denticola can penetrate into the periodontal tissue where it activates inflammatory cells and releases cytopathic and hemolytic factors which can aggravate the tissue destruction (Fenno and McBride, 1998; Fenno, 2012). T. denticola is also designated as a pathobiont taking a role in the destructive inflammatory responses after homeostasis has already disrupted (Hajishengallis, 2014).

Periodontal pathogens are capable of causing massive destruction directly by invading the tissue and producing damaging substances (Taichman and Lindhe, 1990). A set of molecules derived from pathogens, known as pathogen-associated molecular patterns (PAMPs), recognized by host inflammatory cells via Pattern Recognition Receptors (PRRs) such as Toll-like receptors (TLRs), can induce inflammatory responses. This response may also contribute to tissue destruction. In addition to periodontal pathogens, some cell-derived molecules can also react as danger signals and activate inflammatory responses. Such molecules are termed as endogenous damage-associated molecular patterns (DAMPs) or alarmins, and these molecules primarily play a role in normal cell functions (Calogero et al, 1999). During cellular damage, they can act as proinflammatory cytokines, activate PRRs, and stimulate inflammatory response (Tang et al, 2012).

High mobility group box 1 (HMGB1) is an endogenous molecule which normally facilitates cell transcription, but can also act as a DAMP (Yamada and Maruyama, 2007). It can be released during cellular stress mainly after infection or cell necrosis/apoptosis (Qin et al, 2006; Tang et al, 2012). HMGB1, as a DAMP, can recruit inflammatory cells and promote the secretion of proinflammatory cytokines. This multimolecular up-regulated cascade is mediated by several receptors, some of those shared with PAMPs (Kang et al, 2014). The most common HMGB1 receptors are RAGE, TLR2, TLR4, and TLR9 (Park et al, 2006).

Evidence suggests that apoptosis and necrosis might play a significant role in the development of chronic inflammatory process and are associated with tissue destruction in periodontitis (Bantel et al, 2005; Abuhussein et al, 2013; Tunali et al, 2014). Although some apoptosis could occur in the clinically healthy gingiva, especially in the junctional epithelium, a phenomenon that may play a role in the regulation of mucosal inflammation for the maintenance of homoeostasis, increased apoptosis and reduced proliferation observed in the deepest sulcular pocket and most apical part of the pocket might be an essential factor in progression of periodontitis (Tonetti et al, 1998; Jarnbring et al, 2002). Periodontopathogens are associated with cell apoptosis and necrosis with concomitant activation of the immune system (Uitto et al, 1995; Leung et al, 2002; Kelk et al, 2011). The various inflammatory processes could also drive surrounding tissue cells, granulocytes and macrophages to apoptosis and thus increase and maintain the inflammation (Haanen and Vermes, 1995). The release of proinflammatory cytokines and DAMPs by necrotic cells can induce apoptosis and the subsequent increase of the apoptotic cells could lead to secondary necrosis in periodontitis as reported in some 
other inflammatory diseases (Qin et al, 2006; Al-Samadi et al, 2015). However, the exact mechanisms how apoptosis might cause the inflammation and destruction in periodontitis remain unclear.

We aimed to determine the presence of several markers associated with T. denticola, apoptosis, HMGB1, and proinflammatory cytokines and cells in gingival tissue specimens from periodontitis patients in comparison with specimens from gingivitis patients. We hypothesized that there is an association between these factors and the inflammatory process in periodontitis which could be driven by HMGB1.

\section{Materials and Methods}

\section{Patients and samples}

Biopsy samples from patients with periodontitis ( $\mathrm{n}=10$; age range $38-76$, mean 54,0 years; 5 males and 5 female, 9 Finnish and 1 Indonesian) or gingivitis (n=5; age range 19-23, mean 20,4 years; 4 males and 1 female, 5 Indonesian) were used. Periodontitis was diagnosed by clinical analysis of pocket depths, loss of attachment, bone loss, and bleeding on probing. The periodontitis patients had radiographic evidence for bone loss of 20$50 \%$ on many teeth and loss of attachments ranging from 4-6 mm. Periodontitis samples were obtained from premolar-molar regions during flap surgery of the initial periodontal therapy. The patients did not receive any antimicrobial therapy or professional periodontal treatment to the sampling area prior to operation (Rautemaa et al, 2004). Gingivitis was defined with the presence of redness, swelling, and bleeding on probing (Gölz et $a l$, 2014). Gingivitis samples for this study were obtained from patient with gingival index $<2$ and probing depth $\leq 4 \mathrm{~mm}$, showing no supporting tissue destruction and recovered during gingivectomy in the case of gingival enlargement in premolar, canine, or incisor site. All procedures were done under local anesthesia. All samples were formalin-fixed and paraffin-embedded (FFPE). Tissue sections were evaluated by oral pathologists. Periodontitis tissue samples contained the oral, sulcular, and junctional epithelium and beneath that the lamina propria and gingivitis tissue samples contained the oral and sulcular epithelium and the lamina propria. The study was conducted in accordance with the Declaration of Helsinki and was approved by the Ethical Committees of Institute of Dentistry, University of Helsinki (Järvensivu et al, 2004, Rautemaa et al, 2004) and Faculty of Dentistry, Universitas Gadjah Mada, Indonesia. All participants gave their informed consent after they were explained about the study protocol.

\section{Immunostaining}

All antibodies used were commercially available except $T$. denticola CTLP antibody (Supplementary Table 1). T. denticola CTLP antibody was prepared as follows. The protease was first purified from an extract obtained by sonication of $T$. denticola ATCC 35405 cells as done by Uitto et al (1988) and then used to immunize a New Zealand white rabbit (Grenier et al, 1990). The immunoglobulin G (IgG) fraction was purified by passing the antiserum through a column of protein A-Sepharose CL 4B (Sigma Chemical Co., St. Louis, Mo.), eluted with $0.1 \mathrm{M}$ glycine-0.5 M NaCl buffer ( $\mathrm{pH} 2.5$ ), and dialyzed against $50 \mathrm{mM}$ PBS.

All FFPE tissue samples were cut into $4 \mu \mathrm{m}$ sections. The method for immunofluorescence staining of caspase3, HMGB1, TLR-4, CD-68, CD-8, mast cell tryptase (MCT) and immunohistochemistry of TLR-4, IL-1 $\beta$, and IL-8 were done according to previous protocols (Al-Samadi et al, 2014). The immunohistochemistry staining method of T. denticola CTLP was modified from Järvensivu et al (2004). Briefly, tissue sections were 
deparaffinized in citrate buffer using a microwave oven (MicroMED T/T Mega Histoprocessing Labstation; Milestone Srl, Sorisole, Italy) and then incubated with $0.3 \% \mathrm{H}_{2} \mathrm{O}_{2}$ in PBS for $30 \mathrm{~min}$. After three washes with PBS, slides were incubated in appropriate normal serum (1:50 in 2\% PBS-BSA) provided in the staining kit (Vectastain Elite ABC kit; Vector Laboratories, Burlingame, CA, USA) for $1 \mathrm{~h}$ at room temperature (RT). Next, the sections were incubated with the purified $T$. denticola CTLP antibody (1:3000 in 1\% PBS-BSA) for 30 min at $37^{\circ} \mathrm{C}$ and then kept overnight at $4{ }^{\circ} \mathrm{C}$ in a humid chamber. After three washes, the sections were incubated with the corresponding biotinylated secondary antibody (1:200 in 0.1\% PBS-BSA) for 30 min at $37^{\circ} \mathrm{C}$. After washes in PBS, slides were incubated in avidin-biotin-peroxidase complex for $30 \mathrm{~min}$ at $37^{\circ} \mathrm{C}$ and color was developed using $0.006 \% \mathrm{H}_{2} \mathrm{O}_{2}$ substrate and $0.023 \%$ 3,3'-diaminobenzidine tetrahydrochloride (DAB) chromogen for 10 min at RT. The slides were washed with tap water and nuclei were counterstained with Mayer's hematoxylin for $4 \mathrm{~min}$, and then rinsed with tap water. Finally, slides were dehydrated and mounted with Pertex (HistoLab, Gothenburg, Sweden). Negative controls were performed with non-immune species specific IgGs and for pathogen markers by omitting the primary antibody. T. denticola CTLP staining method and sensitivity were done according to previous studies (Marttila et al, 2014). T. denticola was detected by identifying the presence of CTLP intracellularly in the epithelium and extracellularly in the lamina propria.

\section{TUNEL Staining}

Terminal TdT-mediated dUTP-biotin nick end labeling (TUNEL) method assessed the presence of deathassociated DNA fragmentation in situ and was performed using DeadEnd Colorimetric TUNEL System kit (Promega ${ }^{\circledR C}$ Corporation, Fitchburg, WI) according to the manufacturer's protocol.

\section{Microscopy and Image Analysis}

The samples were examined with Leica DM6000 light microscope connected to a digital camera (DFC420 and DFC365FX; Leica Microsystems, Wetzlar, Germany). ImageJ program (Wayne Rasband, National Institute of Mental Health, Bethesda, MD, USA) was used to calculate the expression of HMGB1. The total number of all cells and HMGB1-positive cells were automatically calculated with ImageJ from three different microscope fields at 400x magnification in the lamina propria of each sample. Subcellular localization of HMGB1 was observed by highlighting the nuclear colocalization of HMGB1 using the colocalization highlighter plugin. Nuclear localization was determined if HMGB1 was only restricted into the nucleus. Cytoplasmic expression was counted by subtracting the nuclear HMGB1-positive cells from total HMGB1-positive cells. The expression of HMGB1 was displayed as a percentage of cytoplas mic and nucleic positive cells from total cells. Caspase-3 expression was calculated per ten CD68 positive cells at 400x magnification.

\section{Statistical Analysis}

The data were analyzed statistically using IBM SPSS Statistic 21 software. Paired t-test was used to calculate the mean difference between HMGB1 expression in the cytoplasm and nucleus in each group. Between groups, the mean differences of HMGB1 expression were calculated using the independent t-test and the percentage of caspase-3 in CD68 positive cells using One-way ANOVA followed by post hoc Tukey test. The correlation between age differences was analyzed using a Spearman's correlation. P values less than or equal to 0.05 were considered as statistically significant. 


\section{Results}

\section{Periodontitis samples infected by Treponema denticola}

T. denticola CTLP was detected in tissue samples from periodontitis patients. CTLP positive staining was localized intracellularly in the sulcular epithelium (Figure 1a-c) and was specifically distributed from superficial (upper epithelium layer) to the deeper layer of the epithelium. This typical staining pattern, indicating the invasion and penetration of CTLP, has been shown in earlier studies (Uitto et al, 1995; Marttila et al, 2014). CTLP could additionally be found extracellularly only in periodontitis samples, being present in subgingival plaque and in granular deposits in lamina propria (Figure 1d-f).

\section{Caspase-3 and CD68 Increased in Periodontitis}

We used a cleaved caspase-3 antibody to specifically identify the active form. Although caspase-3 positive cells were present in gingivitis, their number increased in periodontitis. In gingivitis and periodontitis, caspase3 was expressed mainly in the cells of lamina propria and to less extent in the sulcular epithelium (Figure 2a).

CD68 positive cells are professional phagocytic cells which exhibit a high rate and a capacity for phagocytosis and has the main role in apoptotic clearance to avoid the release of DAMPs from apoptotic cells (Zhu et al, 2009; Elliott and Ravichandran 2010; Davidovich et al, 2014). To investigate the expression of caspase-3 on macrophages, double immunofluorescence staining of caspase-3 in a combination with CD68 as a macrophage marker was used (Figure 2a). CD68 positive cells were mostly observed in the lamina propria and only a few were detected in the epithelium. CD68 expression in the epithelial cells is possible during infection or after oxidative stress as reported by Travaglione et al (2002) and Tanaka et al (2008). The percentage of CD68 positive cells that expressed caspase-3 were significantly increased mainly in the lamina propria in periodontitis compared to gingivitis (Figure 2b). The correlation analyses indicated that there were no significant difference between the percentage of caspase-3 positivity in CD68 positive cells with the age of periodontitis patients $(\mathrm{p}=0.986)$ and gingivitis patients $(\mathrm{p}=0.260)$.

\section{TUNEL Positive Cells Increased in Periodontitis both in Epithelial and Lamina Propria}

We confirmed the apoptosis by detecting the presence of cells with death-associated DNA fragmentation in situ by TUNEL labeling (Gamonal et al, 2001). We found a similar pattern between TUNEL labeling and caspase-3 staining, which confirmed the high level of apoptotic cells found in periodontitis (Figure 2a). In periodontitis, TUNEL-positive cells were present in oral, sulcular, and junctional areas with the majority in the lamina propria beneath the junctional epithelium (Supplementary Figure 1).

\section{HMGB1 is Widely Expressed in Periodontitis}

HMGB1 was detected in the nucleus and cytoplasm of cells in the oral epithelium and lamina propria for all samples. In the sulcular epithelium, HMGB1 was mostly expressed in the basal layer and more concentrated in the nucleus in gingivitis and diffused in the cytoplasm in periodontitis samples (Figure 3a). In the junctional epithelium of periodontitis specimens, HMGB1 was expressed in all layers of the epithelium both in the nucleus and cytoplasm (Supplementary Figure 1).

In the lamina propria, HMGB1 was expressed in inflammatory infiltrates and fibroblasts (Figure 3a) and to a lesser extent extracellularly. The number of HMGB1 positive cells in the lamina propria was significantly 
increased in periodontitis compared with gingivitis (Figure 3b). HMGB1 expression in the cytoplasm was significantly higher compared to nuclear expression in periodontitis, whereas there was no significant difference in gingivitis. The cytoplasmic expression of HMGB1 in periodontitis was also significantly higher compared to gingivitis (Figure 3c). The extracellular release of HMGB1 was expressed more in periodontitis than in gingivitis.

\section{TLR4 Expression was Significantly Higher in Periodontitis}

In the oral epithelium of gingivitis, TLR4 was more condensed in the basal and suprabasal layer (the epithelial layer between basal and superficial). The staining intensity gradually became weaker in the upper layer (Figure 4). The sulcular epithelium of periodontitis showed a different staining pattern compared with gingivitis. In the oral epithelium of periodontitis samples, all layers expressed TLR4 with almost similar intensity. In the sulcular and junctional epithelium, all layers expressed TLR4 also with similar intensity but the intensity was slightly weaker compared with the oral epithelium (Supplementary Figure 1).

In the lamina propria of all groups, many types of cells expressed TLR4, including fibroblasts, endothelial, and some inflammatory cells. TLR4-positive cells greatly increased in periodontitis compared to gingivitis.

To investigate the types of cells that express TLR4, we performed a double immunofluorescence staining technique using TLR4 labeling in combination with a marker for macrophages (CD68), mast cells (MC Tryptase), and T-cells (CD8). TLR4 was expressed by macrophages, rarely by mast cells, and mostly by CD8 T-cells in periodontitis (Figure 4).

\section{Proinflammatory Cytokines Increased in Periodontitis}

Immunohistochemical staining of IL-8 and IL-1 demonstrates that both were increased in the sulcular epithelium of periodontitis compared with the sulcular epithelium of gingivitis (Figure 5). In gingivitis, the staining intensity of IL- 8 and IL-1 $\beta$ in the sulcular epithelium was increased as well as the number of the positive cells in the lamina propria. In periodontitis, the staining of IL- 8 and IL- $1 \beta$ was stronger in the junctional compared with the sulcular and oral epithelium, and also in the lamina propria, the number of positive cells were increased as well as the extracellular expression of IL-8 and IL-1 $\beta$ (Supplementary Figure $1)$.

\section{Discussion}

Immunohistochemical results of this study confirm that the presence of $T$. denticola (especially its CTLP), apoptotic cells (especially macrophages), HMGB1, TLR4 (HMGB1 receptor), and proinflammatory cytokines were significantly increased in periodontitis compared to gingivitis. These results support the hypothesis that the inflammatory process mediated by HMGB1, one of the danger signal molecules, might occur in periodontitis.

This study comprises of relatively small sample size, which may limit the general applicability of our results. Differential age distribution might also affect the results; however, the correlation analyses indicated that the correlation between the age and the calculated apoptotic cells was not statistically significant. The biopsy samples were also carefully selected according to specific criteria followed by the evaluation based on 
histological findings. Lack of classification of the disease severity in periodontitis might also lead to misinterpretation of the outcome which will more generalize the mechanism in all stages of periodontitis.

The inflammation process observed during periodontitis is initiated by periodontopathogens, most likely by members of the red complex. (T. denticola, Porphyromonas gingivalis, and Tannerella forsythia), which have been strongly associated with the severity of periodontitis (Socransky et al, 1998). However, endogenous DAMPs or alarmins which are found in several chronic inflammatory diseases may also be involved in the pathogenesis of periodontitis. DAMPs are produced or induced by the cells which undergo apoptosis, necroptosis or necrosis (Shi et al, 2003; Bell et al, 2006; Cullen et al, 2013; Davidovich et al, 2014).

We showed that all periodontitis tissue samples were infected by $T$. denticola. Increasing information indicates that infection of tissues by some pathogenic bacteria indeed triggers host cell apoptosis (Zychlinsky and Sansonetti, 1997; Lancellotti et al, 2006). Although apoptosis has a critical role in eliminating harmful or injured cells during inflammation, infection-induced apoptosis also can promote the proinflammatory process (Zychlinsky and Sansonetti, 1997). The formation of biofilm and the high amount of the apoptotic cells in the area of the junctional epithelium in periodontitis might occur also from natural turnover and thus could be related to inflammation. So far, even though several research studies have reported that apoptosis is increased in periodontitis and suggested its possible role in promoting inflammation, its effect has not yet been clearly elucidated. Here we brought evidence for a possible connection between apoptosis, DAMPs, and inflammation in periodontitis.

The increase of caspase-3 and TUNEL-positive cells in the periodontitis samples in our study showed that most cells in periodontitis were undergoing apoptosis. The apoptotic cells were predominantly present in the sulcular epithelium and junctional epithelium and in the lamina propria beneath it, in the area where the pathogens are invading into and accumulating in the tissue (Kornman et al, 1997). Interestingly, apoptosis was also slightly increased in the oral epithelium and the lamina propria beneath it, although the area was further away from the pathogen infected site. This result demonstrates that apoptosis in periodontitis could be induced directly by the pathogens in the sulcular and junctional area and also indirectly by the host immune reaction in the oral area. This could be, at least in part, caused by $T$. denticola CTLP since it was reported to have a cytopathic effect, which cause apoptosis in epithelial and endothelial cells (Uitto et al, 1995; Bernardini et al, 2010). The overall high apoptosis in the sub epithelium of lamina propria in this study may show that inflammatory infiltrates and fibroblasts were rapidly going into apoptosis by the influence of pathogens and their products such as proteinases which promote apoptosis in fibroblasts (Pöllänen et al, 2012). The higher number of apoptotic macrophages in periodontitis might be induced by $T$. denticola as reported by Jun et al (2017). The increase of apoptotic macrophages (as professional phagocytes) with the excess of apoptotic cells in periodontitis may lead to failure of efficient clearance of apoptotic cells. The membrane integrity of untreated apoptotic cells will be lost and the cells progress to secondary necrosis, release intracellular content including HMGB1, and induce cytokine production (Green et al, 2009; Elliott and Ravichandran, 2010). T. denticola-induced macrophage cell death itself will release DAMPs (Jun et al, 2017) and in vice versa, the certain dose of HMGB1 could induce apoptosis in macrophages (Zhu et al, 2009). This positive loop will increase the severity of the inflammation as found in several diseases such as chronic lung disease, neuropathy, atherosclerosis, and arthritis (Velegraki et al, 2013; Grabiec and Hussell, 2016). 
Besides necrotic and activated cells, some studies indicate that apoptotic cells could also change nuclear localization and release of HMGB1 (Bell et al, 2006; Abdulahad et al, 2013). Apoptotic cells lead to active secretion of HMGB1 by macrophages, as do macrophages engulfing apoptotic cells (Qin et al, 2006; Jiang et $a l$, 2007). In our staining, we observed an increased change in the expression pattern of HMGB1 in periodontitis both in the epithelium and in the lamina propria, and also found some extracellular HMGB1 in periodontitis tissue. The infiltration of inflammatory cells was higher in periodontitis compared to gingivitis and may affect the incidence of apoptotic and $\mathrm{HMGB} 1^{+}$cells, thus increasing the secretion of HMGB1 in periodontitis. Luo et al (2011) also reported the increased release of HMGB1 in gingival crevicular fluid. This may suggest that the increase of apoptotic cells in periodontitis leads to changes in HMGB1 expression through the release of HMGB1 by themselves and also phagocytic clearance of apoptotic cells by macrophages that will be activated to HMGB1 release. The shift of HMGB1 translocation from the nucleus to the cytoplasm and subsequent secretion suggests that HMGB1 is extensively acetylated and phosphorylated (Youn and Shin, 2006). A minor release of HMGB1 from apoptotic cells will not be recognized as a proinflammatory signal since it undergoes oxidative modification and delivers tolerogenic signals which suppress immune activity (Carta et al, 2009; Sims et al, 2010).

The expression of one of the HMGB1 receptors, TLR4, was affected in periodontitis epithelium and increased in the lamina propria in a parallel manner to that of HMGB1 expression. Other studies have reported the increase of TLR4 expression with LPS in periodontitis (Mori et al, 2003). HMGB1-TLR4 signaling in gingival epithelium might stimulate IL-8 secretion as in nasal epithelium (Shimizu et al, 2016). HMGB1-TLR4 signaling in T-cells has a different effect on cytokine release, proliferation, and Th1 polarization (Messmer $e t$ al, 2004; Li et al, 2013; Kang et al, 2014) while in monocytes/macrophages it stimulates the release of several pro-inflammatory cytokines, such as IL-1 $\beta$, IL-8, and TNF- $\alpha$ (Andersson et al, 2000; Yu et al, 2006).

The increase of IL-1 $\beta$ and IL-8 will also increase the severity of periodontal diseases (Hou et al, 2003). Smoking may also affect the production of these inflammatory factors, however the studies related to this aspect are contradictory (Gonçalves et al, 2011). IL-1 $\beta$ and IL-8 contribute to the recruitment of additional neutrophils, promote Th17 polarization (van de Veerdonk et al, 2011), and stimulates several other cell types to produce soft tissue catabolic and bone resorptive-mediators which induce tissue destruction in periodontitis (Genco, 1992; Boch et al, 2001).

This study shows that the presence of periodontopathogen infection in periodontitis was accompanied by higher expression of apoptosis, HMGB1, TLR4, and proinflammatory cytokines compare to gingivitis. We conclude that the periodontopathogen infection may associate with the increase of apoptosis, DAMPs, and inflammation in periodontitis. However, we have to keep in mind that this conclusion is based only on immunohistological data with a relatively small sample size and therefore, further investigations that overcome these study drawback and in vitro studies are needed to trace and clearly elucidate the mechanism of this pathway followed by in vivo intervention trials in animals.

\section{Acknowledgements}

This study was supported by Directorate General of Human Resource for Science, Technology and Higher Education of Indonesia, Center for International Mobility (CIMO), Jane and Aatos Erkko Foundation, Selma and Maja-Lisa Selanders fund, Otto A. Malm Foundation, Sigrid Jusélius Foundation, grants from the Helsinki 
University Hospital Research Foundation, Helsinki, Finland and the Karolinska Institutes, Stockholm, Sweden. Dr. Anne Järvensivu, DDS. is acknowledged for providing the tissue samples. Conflicts of interest: none to declare.

\section{References}

Abdulahad DA, Westra J, Reefman E, et al (2013). High mobility group box1 (HMGB1) in relation to cutaneous inflammation in systemic lupus erythematosus (SLE). Lupus 22: 597-606.

Abuhussein H, Bashutski JD, Dabiri D, et al (2014). The role of factors associated with apoptosis in assessing periodontal disease status. J Periodontol 85: 1086-1095.

Al-Samadi A, Salem A, Ainola M, Hietanen J, Häyrinen-Immonen R, Konttinen YT (2015). Increased beta 2 defensin in recurrent aphthous ulcer. Oral Dis 21: 292-298.

Al-Samadi A, Drozd A, Salem A, Hietanen J, Hayrinen-Immonen R, Konttinen YT (2015). Epithelial cell apoptosis in recurrent aphthous ulcers. J Dent Res 94: 928-935.

Andersson U, Wang H, Palmblad K, et al (2000). High mobility group 1 protein (HMG-1) stimulates proinflammatory cytokine synthesis in human monocytes. J Exp Med 192: 565-570.

Bantel H, Beikler T, Flemmig TF, Schulze-Osthoff K (2005). Caspase activation is involved in chronic periodontitis. FEBS Lett 579: 559-564.

Bell CW, Jiang W, Reich CF, Pisetsky DS (2006). The extracellular release of HMGB1 during apoptotic cell death. Am J Physiol 291: C1318-1325.

Bernardini C, Gaibani P, Zannoni A, et al (2010). Treponema denticola alters cell vitality and induces HO-1 and Hsp70 expression in porcine aortic endothelial cells. Cell Stress Chaperones 15: 509-516.

Boch JA, Wara-aswapati N, Auron PE (2001). Interleukin 1 signal transduction--current concepts and relevance to periodontitis. J Dent Res 80: 400-407.

Calogero S, Grassi F, Aguzzi A, et al (1999). The lack of chromosomal protein Hmg1 does not disrupt cell growth but causes lethal hypoglycaemia in newborn mice. Nat Genet 22: 276-280.

Carta S, Castellani P, Delfino L, Tassi S, Venè R, Rubartelli A (2009). DAMPs and inflammatory processes: the role of redox in the different outcomes. J Leukoc Biol 86: 549-55.

Cullen SP, Henry CM, Kearney CJ, et al (2013). Fas/CD95-induced chemokines can serve as 'Find-Me' signals for apoptotic cells. Mol Cell 49: 1034-1048.

Davidovich P, Kearney CJ, Martin SJ (2014). Inflammatory outcomes of apoptosis, necrosis and necroptosis. Biol Chem 395: 1163-1171.

Elliott MR, Ravichandran KS (2010). Clearance of apoptotic cells: Implications in health and disease. J Cell Biol 189: 1059-1070. 
Fenno JC (2012). Treponema denticola interactions with host proteins. J Oral Microbiol 4: 9929.

Fenno JC, McBride BC (1998). Virulence factors of oral treponemes. Anaerobe 4: 1-17.

Gamonal J, Bascones A, Acevedo A, Blanco E, Silva A (2001). Apoptosis in chronic adult periodontitis analyzed by in situ DNA breaks, electron microscopy, and immunohistochemistry. J Periodontol 72: 517-525.

Genco RJ (1992). Host responses in periodontal diseases: current concepts. J Periodontol 63: 338-355.

Green DR, Ferguson T, Zitvogel L, Kroemer G (2009). Immunogenic and tolerogenic cell death. Nat Rev Immunol 9: 353-363.

Grenier D, Uitto VJ, McBride B (1990). Cellular location of a Treponema denticola chymotrypsinlike protease and importance of the protease in migration through the basement membrane. Infect Immun 58: 347-351.

Gonçalves RB, Coletta RD, Silvério KG, Benevides L, Casati MZ, da Silva JS, Nociti FH (2011). Impact of smoking on inflammation: overview of molecular mechanisms. Inflamm Res 60: 409-424.

Gölz L, Memmert S, Rath-Deschner B, et al (2014). LPS from P. gingivalis and hypoxia increases oxidative stress in periodontal ligament fibroblasts and contributes to periodontitis. Mediators Inflamm 2014: 1-13.

Haanen C, Vermes I (1995). Apoptosis and inflammation. Mediators Inflamm 4: 5-15.

Hajishengallis G (2014). Immunomicrobial pathogenesis of periodontitis : keystones, pathobionts , and host response. Trends Immunol 35: 3-11.

Hou L-T, Liu C-M, Liu B, Lin S, Liao C-S, Rossomando EF (2003). Interleukin-1beta, clinical parameters and matched cellular-histopathologic changes of biopsied gingival tissue from periodontitis patients. J Periodontal Res 38: $247-254$.

Jarnbring F, Somogyi E, Dalton J, Gustafsson A, Klinge B (2002). Quantitative assessment of apoptotic and proliferative gingival keratinocytes in oral and sulcular epithelium in patients with gingivitis and periodontitis. J Clin Periodontol 29: 1065-1071.

Jiang W, Bell CW, Pisetsky DS (2007). The relationship between apoptosis and high-mobility group protein 1 release from murine macrophages stimulated with lipopolysaccharide or polyinosinic-polycytidylic acid. $J$ Immunol 178: 6495-6503.

Jun H, Jung Y, Choi B (2017). Treponema denticola, Porphyromonas gingivalis, and Tannerella forsythia induce cell death and release of endogenous danger signals. Arch Oral Biol 73: 72-78.

Kang R, Chen R, Zhang Q, et al (2014). HMGB1 in health and disease. Mol Aspects Med 40: 1-116.

Kelk P, Abd H, Claesson R, Sandström G, Sjöstedt A, Johansson A (2011). Cellular and molecular response of human macrophages exposed to Aggregatibacter actinomycetemcomitans leukotoxin. Cell Death Dis 2 : e126. 
Kornman KS, Page RC, Tonetti MS (1997). The host response to the microbial challenge in periodontitis: assembling the players. Periodontol 2000 14: 33-53.

Lancellotti M, Brocchi M, Silvedeira W Da (2006). Bacteria-induced apoptosis: an approach to bacterial pathogenesis. Braz J Morphol Sci 23: 75-86.

Leung WK, Wu Q, Hannam PM, McBride BC, Uitto V-J (2002). Treponema denticola may stimulate both epithelial proliferation and apoptosis through MAP kinase signal pathways. J Periodontal Res 37: 445-55.

Li G, Liang X, Lotze MT (2013). HMGB1: The central cytokine for all lymphoid cells. Front Immunol 4: 19.

Luo L, Xie P, Gong P, Tang X, Ding Y, Deng L-X (2011). Expression of HMGB1 and HMGN2 in gingival tissues, GCF and PICF of periodontitis patients and peri-implantitis. Arch Oral Biol 56: 1106-1111.

Marttila E, Järvensivu A, Sorsa $\mathrm{T}$, et al (2014). Intracellular localization of Treponema denticola chymotrypsin-like proteinase in chronic periodontitis. J Oral Microbiol 6: 1-6.

Messmer D, Yang H, Telusma G, et al (2004). High Mobility Group Box Protein 1: An Endogenous Signal for Dendritic Cell Maturation and Th1 Polarization. J Immunol 173: 307-313.

Mori Y, Yoshimura a, Ukai T, Lien E, Espevik T, Hara Y (2003). Immunohistochemical localization of Tolllike receptors 2 and 4 in gingival tissue from patients with periodontitis. Oral Microbiol Immunol 18: 54-58.

Page RC, Offenbacher S, Schroeder HE, Seymour GJ, Kornman KS (1997). Advances in the pathogenesis of periodontitis: summary of developments, clinical implications and future directions. Periodontol 2000 14: 216-248.

Park JS, Gamboni-robertson F, He Q, et al (2006). High mobility group box 1 protein interacts with multiple Toll-like receptors. Am J Am J Physiol Cell Physiol 290: 917-924.

Pöllänen MT, Laine MA., Ihalin R, Uitto V-J (2012). Host-Bacteria crosstalk at the dentogingival junction. Int J Dent 2012: 1-14.

Qin S, Wang H, Yuan R, et al (2006). Role of HMGB1 in apoptosis-mediated sepsis lethality. J Exp Med 203: $1637-1642$.

Rautemaa R, Järvensivu A, Kari K, et al (2004). Intracellular localization of Porphyromonas gingivalis thiol proteinase in periodontal tissues of chronic periodontitis patients. Oral Dis 10: 298-305.

Riviere GR, DeRouen TA (1998). Association of oral spirochetes from periodontally healthy sites with development of gingivitis. J Periodontol 69: 496-501.

Sela MN (2001). Role of Treponema denticola in periodontal diseases. Crit Rev Oral Biol Med 12: 399-413.

Shi Y, Evans JE, Rock KL (2003). Molecular identification of a danger signal that alerts the immune system to dying cells. Nature 425: 516-521. 
Shimizu S, Kouzaki H, Kato T, Tojima I, Shimizu T (2016). HMGB1-TLR4 signaling contributes to the secretion of interleukin 6 and interleukin 8 by nasal epithelial cells. Am J Rhinol Allergy 30: 167-172.

Sims GP, Rowe DC, Rietdijk ST, Herbst R, Coyle AJ (2010). HMGB1 and RAGE in inflammation and cancer. Annu Rev Immunol 28: 367-388.

Socransky SS, Haffajee AD, Cugini MA, Smith C, Kent RL (1998). Microbial complexes in subgingival plaque. J Clin Periodontol 25: 134-144.

Sorsa T, Tjäderhane L, Konttinen YT, et al (2006). Matrix metalloproteinases: contribution to pathogenesis, diagnosis and treatment of periodontal inflammation. Ann Med 38: 306-21.

Taichman N, Lindhe J (1990). Pathogenesis of Plaque-Associated Periodontal Disease, Textbook of Clinical Periodontology. $2^{\text {nd }}$ edition. Copenhagen. Denmark: Munksgaard, pp.153-190

Tanaka M, Suzuki Y, Shirato I, et al (2008). Tubular epithelial cells have the capacity to transdifferentiate into CD68-positive macrophage-like cells by oxidative stress. Inflamm Res 57: 593-600.

Travaglione S, Falzano L, Fabbri A, Stringaro A, Fais S, Fiorentini C (2002). Epithelial cells and expression of the phagocytic marker CD68: scavenging of apoptotic bodies following Rho activation. Toxicol Vitr 16: 405-411.

Tang D, Kang R, Coyne CB, Zeh HJ, Lotze MT (2012). PAMPs and DAMPs: signal 0s that spur autophagy and immunity. Immunol Rev 249: 158-175.

Tonetti MS, Cortellini D, Lang NP (1998). In situ detection of apoptosis at sites of chronic bacterially induced inflammation in human gingiva. Infect Immun 66: 5190-5195.

Tunali M, Ataoğlu T, Çelik I (2014). Apoptosis: an underlying factor for accelerated periodontal disease associated with diabetes in rats. Clin Oral Investig 18: 1825-1833.

Uitto VJ, Grenier D, Chan ECS, McBride BC (1988). Isolation of a chymotrypsinlike enzyme from Treponema denticola. Infect Immun 56: 2717-2722.

Uitto VJ, Pan YM, Leung WK, et al (1995). Cytopathic effects of Treponema denticola chymotrypsin-like proteinase on migrating and stratified epithelial cells. Infect Immun 63: 3401-3410.

van de Veerdonk FL, Netea MG, Dinarello CA, Joosten LAB (2011). Inflammasome activation and IL-1 $\beta$ and IL-18 processing during infection. Trends Immunol 32: 110-116.

Yamada S, Maruyama I (2007). HMGB1, a novel inflammatory cytokine. Clin Chim Acta 375: 36-42.

Youn JH, Shin J-S (2006). Nucleocytoplasmic shuttling of HMGB1 is regulated by phosphorylation that redirects it toward secretion. J Immunol 177: 7889-7897.

Yu M, Wang H, Ding A, et al (2006). HMGB1 signals through toll-like receptor (TLR) 4 and TLR2. Shock 26: $174-179$. 
Zhu XM, Yao YM, Liang HP, Liu F, Dong N, Yu Y, Sheng ZY (2009). Effect of high mobility group box-1 protein on apoptosis of peritoneal macrophages. Arch Biochem Biophys 492: 54-61.

Zychlinsky A, Sansonetti PJ (1997). Apoptosis as a proinflammatory evet: what can we learn from bacteriainduced cell death? Trends Microbiol 5: 201-204.

\section{Table}

Supplementary Table 1. List of the antibodies used in this study.

\section{Figure legends}

Figure 1. Localization of Treponema denticola chymotrypsin-like proteinase (CTLP) in periodontitis gingival tissue. (a) and (d) are hematoxylin-eosin stainings of the consecutive slides. (b) and (c) show $T$. denticola CTLP expression intracellularly in the sulcular epithelium (white arrow), while in (e) and (f) extracellularly in the lamina propria (black arrow) of periodontitis tissue. Hematoxylin (blue) for nuclear counterstain and scale bar $50 \mu \mathrm{m}$.

Figure 2. Double immunofluorescence staining of caspase-3 (red) and CD68 (green) and TUNEL staining in gingivitis and periodontitis. (a) Caspase- 3 expression is increased in periodontitis as also double-positive cells (yellow). TUNEL staining shows the apoptotic nuclei (black arrow) confirming the high apoptosis in periodontitis. Epithelium marked with $\mathrm{E}$ and its basal lamina with the white or black dotted line. (b) Diagram of the percentage of positive CD68 cells that stained with caspase-3. Data is expressed as a mean \pm standard deviation. ${ }^{* * *} \mathrm{p}<0.001$. DAPI (blue) for nuclear counterstain in immunofluorescence and scale bar $50 \mu \mathrm{m}$.

Figure 3. (a) High Mobility Group Box1 (HMGB1) expression in the sulcular epithelium and the lamina propria of gingivitis and periodontitis. Its expression in the lamina propria is increased in periodontitis. Immunofluorescence staining shows the expression of HMGB1 (green) in the lamina propria. DAPI and hematoxylin (blue) for nuclear counterstain. Colocalization of HMGB1 and nuclei is shown in white, cytoplasmic and extracellular expression of HMGB1 in green, while nuclei are converted into red. (b) The graph of the specific localization of HMGB1 in the nucleus and cytoplasm from total cells in the lamina propria. Scale bar $50 \mu \mathrm{m}$. Data is expressed as a mean \pm standard deviation. $* * \mathrm{p}<0.01, * * * \mathrm{p}<0.001$.

Figure 4. TLR4 expression in the lamina propria and the sulcular epithelium of gingivitis and periodontitis and double staining of TLR4 with CD68, CD8 and mast cell tryptase (MCT) in the gingival tissue. Positivity of TLR4 in fibroblasts are marked with a double arrowhead, in endothelial cells are marked with arrowhead, and in inflammatory cells with single arrows. TLR4 was stained red; CD68, CD8 and MCT green; doublepositive cells are yellow. DAPI and hematoxylin (blue) for nuclear counterstain. Scale bar in immunohistochemistry staining $100 \mu \mathrm{m}$, immunofluorescence $50 \mu \mathrm{m}$.

Figure 5. Immunostaining of IL-8 and IL-1 $\beta$ in the lamina propria and the sulcular epithelium of gingivitis and periodontitis. Hematoxylin (blue) for nuclear counterstain and scale bar $100 \mu \mathrm{m}$.

Supplementary Figure 1. Expression of TUNEL, HMGB1, TLR4, IL-8, and IL-1 $\beta$ in the oral, sulcular and junctional epithelium in periodontitis tissue. Scale bar $100 \mu \mathrm{m}$. 


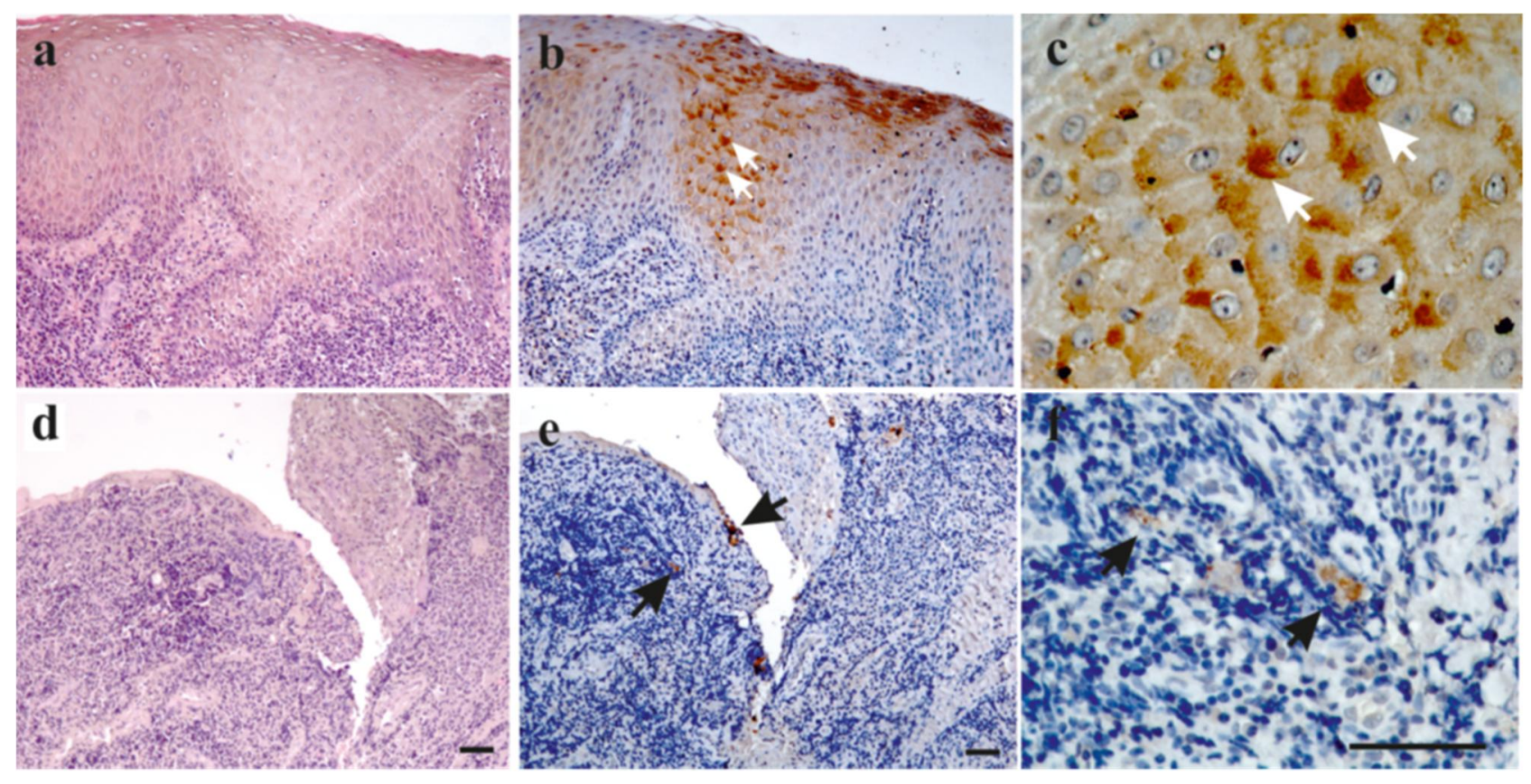


a
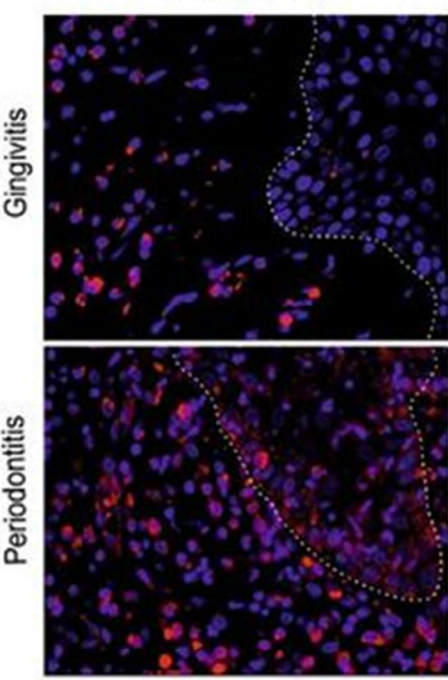

CD68

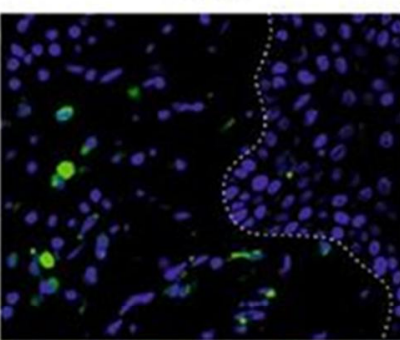

Caspase-3-CD68

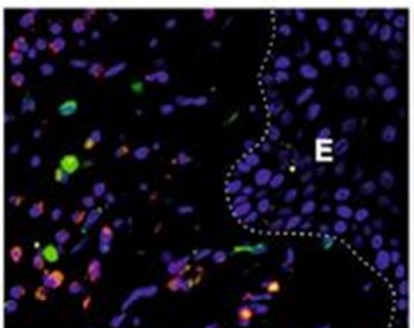

TUNEL

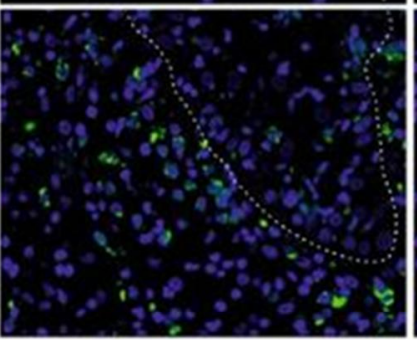

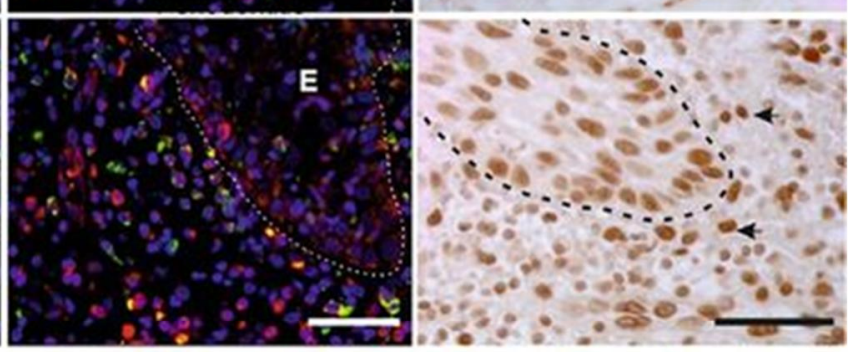

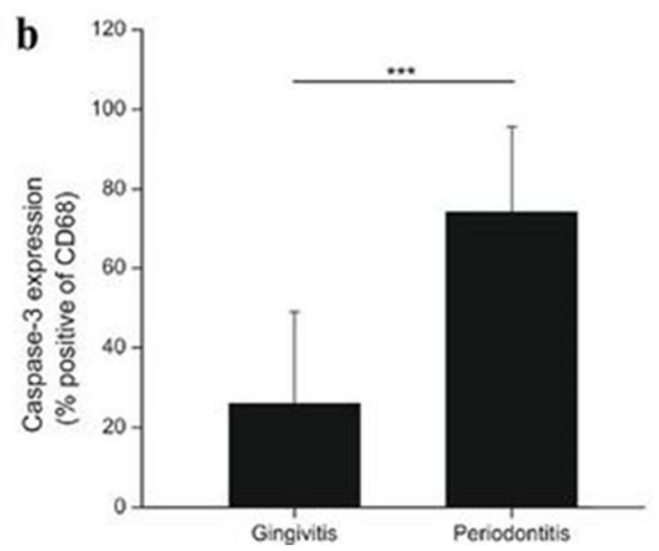



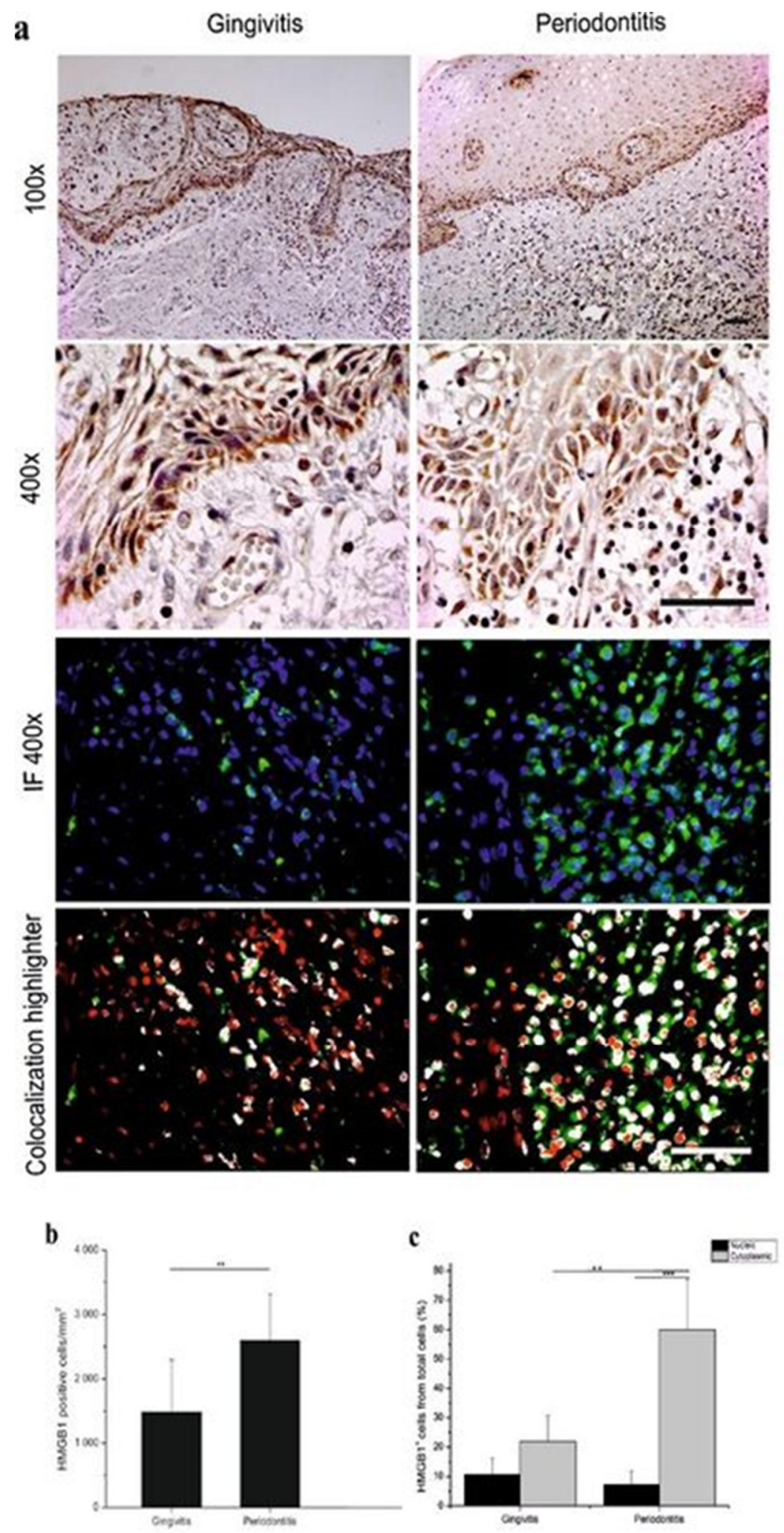
Healthy
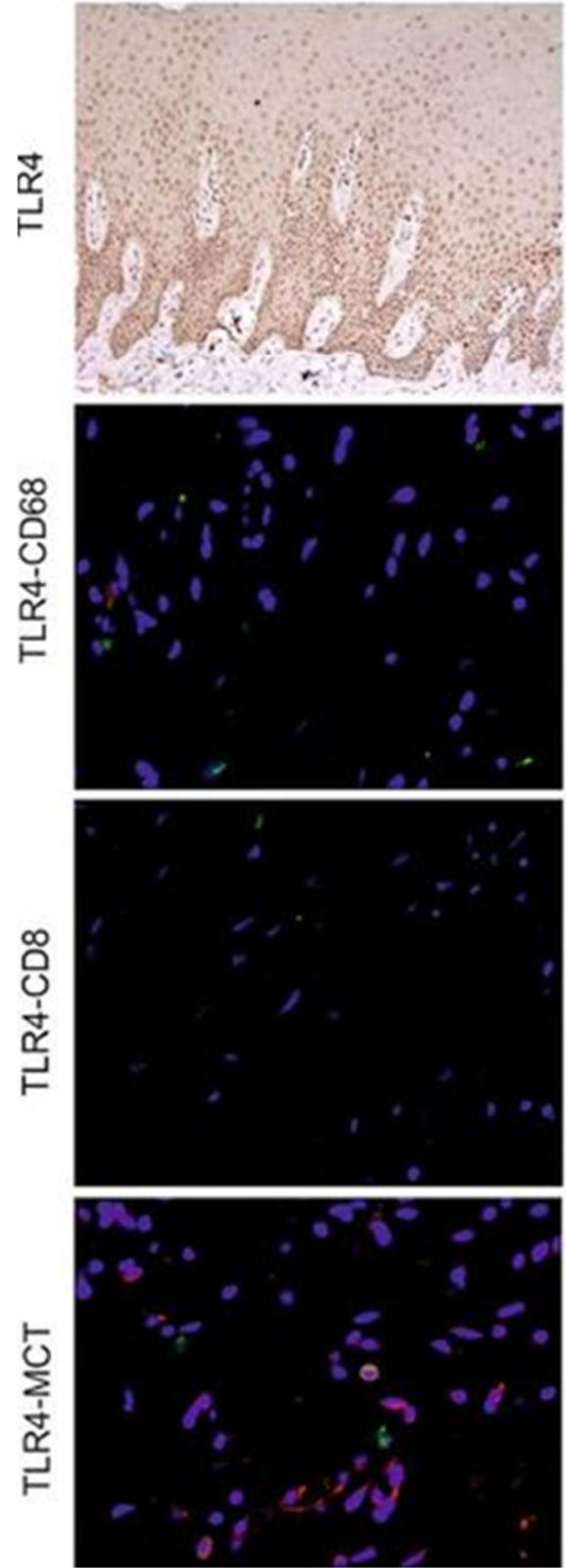

Gingivitis
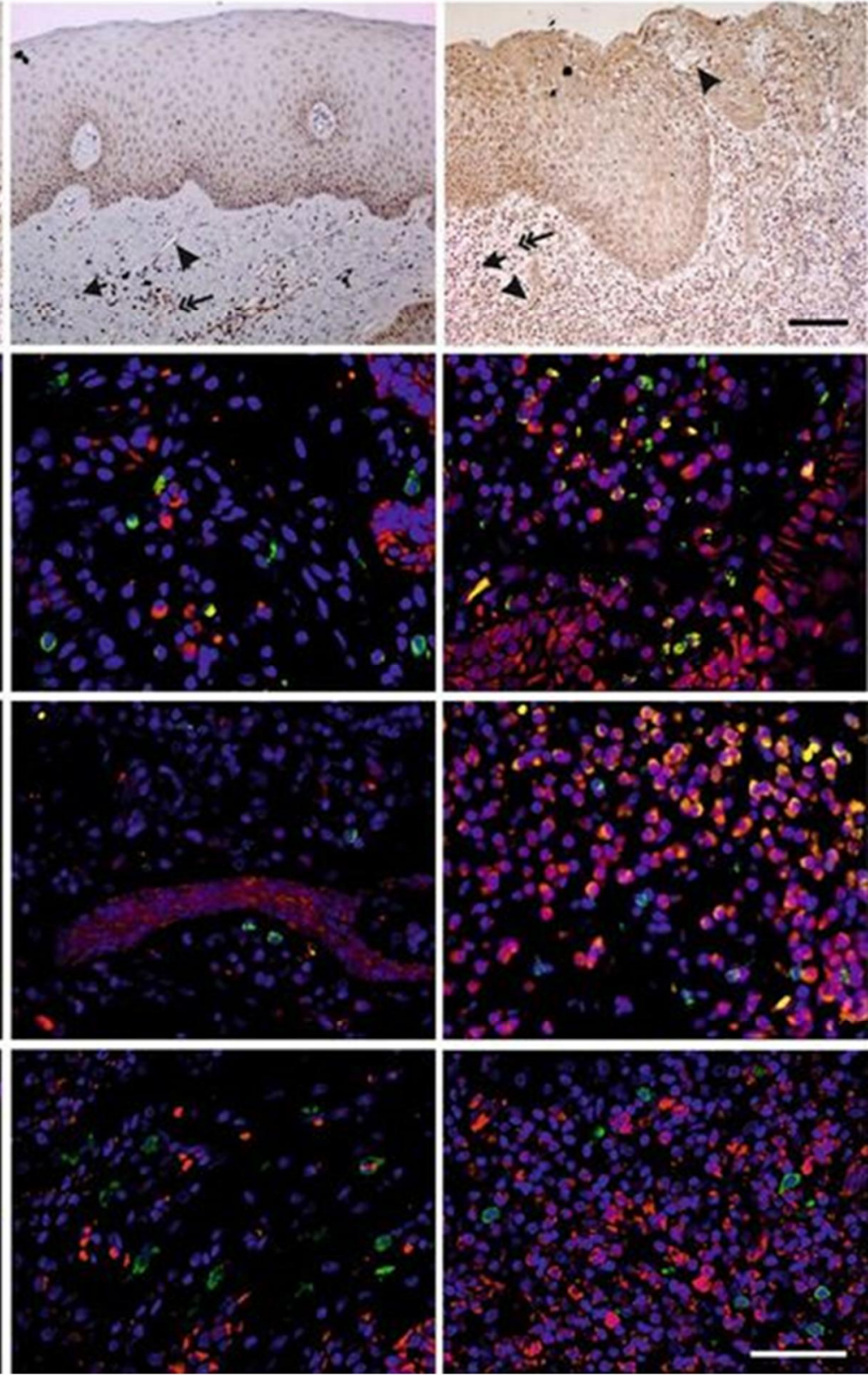

Periodontitis
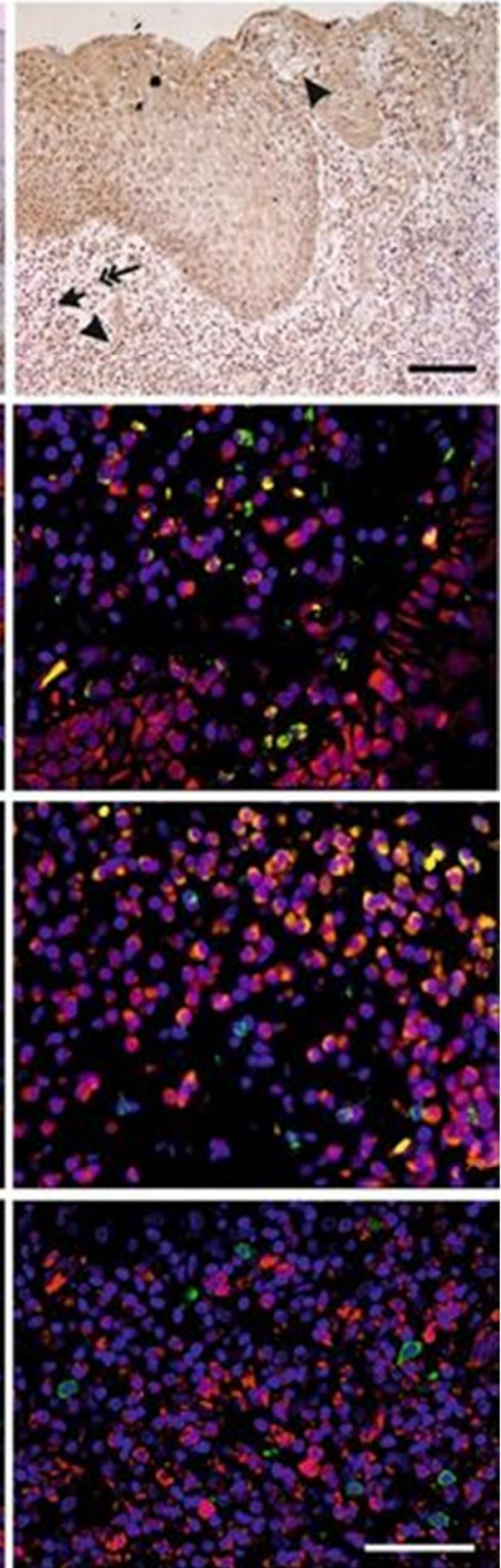

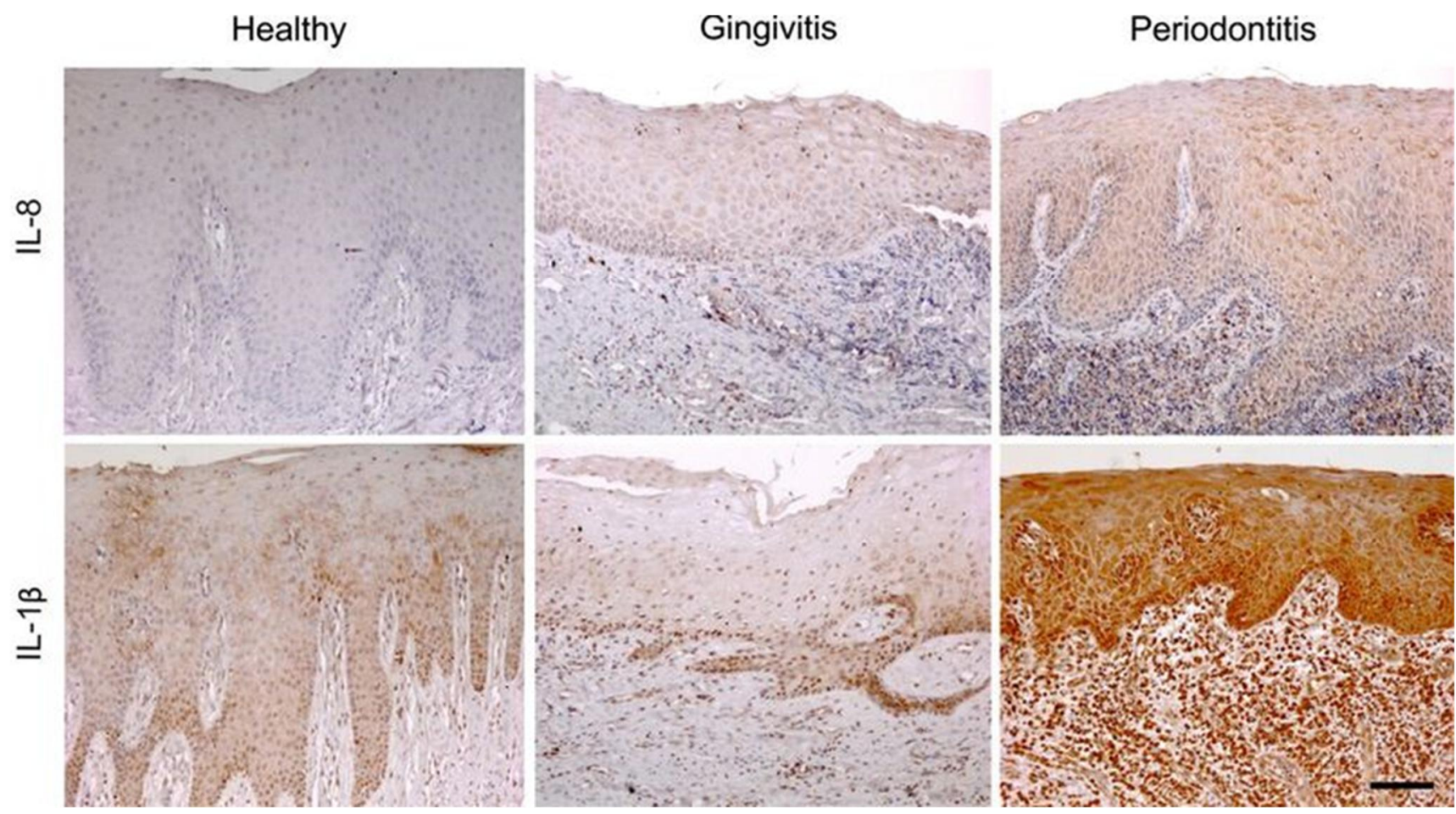


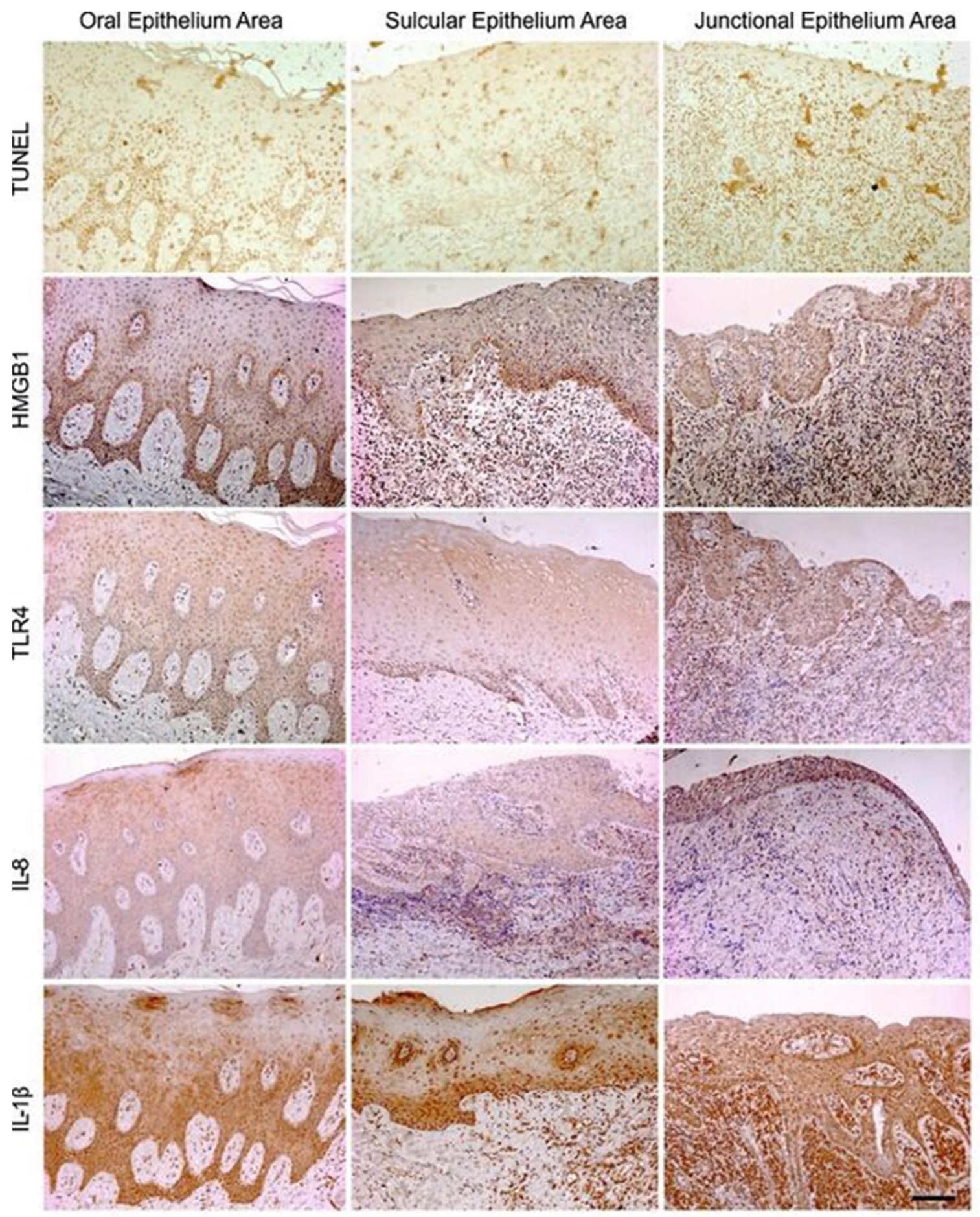

Supplementary Figure 1 . Expression of TUNEL, HMGB1, TLR4, IL-8, and IL- $1 \beta$ in the oral, sulcular and junctional epithelium in periodontitis tissue. Scale bar $100 \mu \mathrm{m}$. 


\section{Control experiments with healthy tissue for TUNEL labelling}

\section{Patients and samples}

Healthy gingival tissues as control samples were obtained during embedded wisdom tooth extraction with no pathology confirmed by clinical examination $(n=5)$. Healthy control samples contained only the oral epithelium and the lamina propria from tissue covering the embedded tooth.

\section{Results}

In healthy controls, only a few cells showed a positive TUNEL staining in the oral epithelium and the lamina propria (Supplementary Figure 2)

Healthy

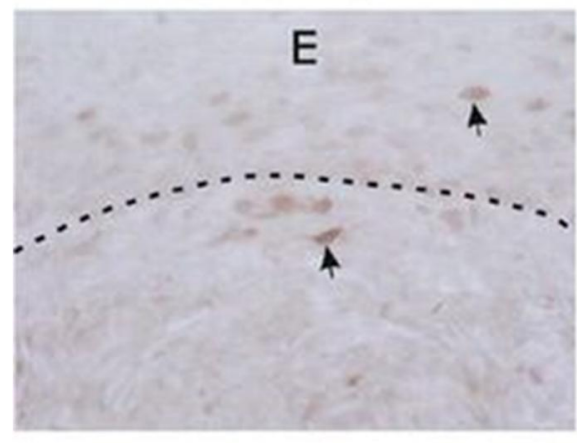

Gingivitis

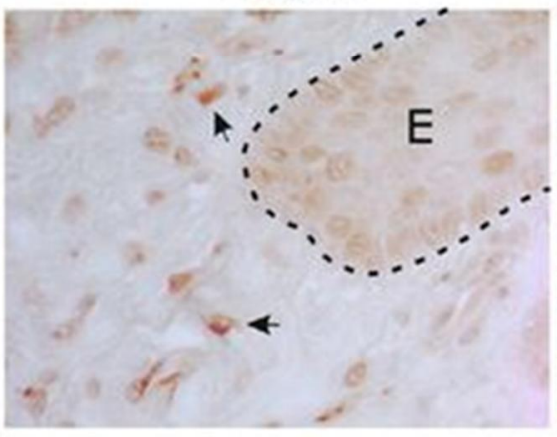

Periodontitis

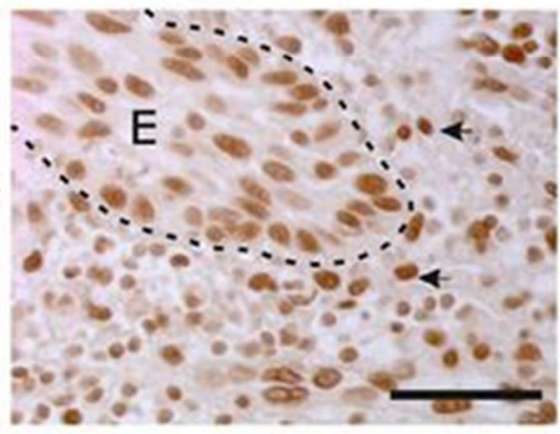

Supplementary Figure 2. TUNEL staining indicates apoptotic cells (arrow) in the epithelium and the lamina propria. Only a few positive cells are located in healthy control samples, more in gingivitis, and highly increased in periodontitis. Epithelium marked with $\mathrm{E}$ and its basal lamina with the black dotted line. Scale bar $50 \mu \mathrm{m}$. 